\title{
RESULTADOS DEL TRATAMIENTO QUIRÚRGICO DE LA PATOLOGÍA PROSTÁtICA BENIGNA EN PACIENTES GERIÁTRICOS.
}

\author{
Marta García Torrelles, Víctor Carrascosa Lloret, Jose Ramón Beltrán Armada, Vicente Rodrigo \\ Guanter, Aleixandre Verges Prosper, Nacho Rubio Tortosa y María Morales Suarez-Varela'.
}

Servicio de Urología. Hospital Universitario Dr. Peset. Valencia.

Departamento de Medicina Preventiva'. Universidad de Valencia. Unidad investigación clínica-epidemiológica Hospital Universitario Dr. Peset. Valencia. España.

\begin{abstract}
Resumen.- OBJETIVO: Valorar el efecto de la edad en la morbi-mortalidad y en el resultado del tratamiento quirúrgico de la hiperplasia benigna de próstata.

MÉTODO: Se ha realizado un estudio retrospectivo, 1999-2003, con una muestra de 305 pacientes mayores de 70 años de edad, con diagnóstico histológico de hiperplasia benigna de próstata intervenidos por resección transuretral de próstata o cirugía abierta. Valorando en cuatro grupos de pacientes, entre 70-80 años de edad y mayores de 80 años de edad para ambas técnicas quirúrgicas, las siguientes variables: ASA, indicación quirúrgica, factores relacionados con la cirugía, la evolución postoperatoria y en el seguimiento. Se ha utilizado como estadísticos el test Anova y $\chi^{2}$ con un nivel de confianza del $95 \%$.
\end{abstract}

RESULTADOS: En nuestra serie se llevó a cabo cirugía abierta en el $59.1 \%$ de los casos y resección transuretral de próstata en el 40.9\%. El 98.4\% de los pacientes presentaba algún grado de comorbilidad. La sonda vesical permanente era la principal indicación quirúrgica en pacientes mayores de 80 años de edad, mientras que el prostatismo severo lo era para los pacientes entre 70-80 años de edad. Las complicaciones urológicas son más frecuentes que las complicaciones generales, $14 \%$ y $10.1 \%$ respectivamente. Las complicaciones urológicas más frecuentes son las menos graves, sobre todo inflamatorio-infecciosas. Además se observa que las complicaciones urológicas y las generales se dan sobre todo en el grupo de cirugía abierta sin encontrar diferencias significativas según el grupo de edad. La satisfacción es elevada en todos los grupos 184.8\%$96.2 \%)$. No ocurrió ningún éxitus perioperatorio, y sólo un paciente falleció en el postoperatorio inmediato.

CONCLUSIÓN: Pensamos que las diferencias observadas no deben atribuirse a la edad cronológica per se entendida como factor de riesgo clínico. Por lo tanto, estos pacientes pueden beneficiarse de un tratamiento quirúrgico resolutivo consiguiendo mejorar su calidad de vida.

Palabras clave: Hiperplasia benigna prostática. Edad. Comorbilidad.

Summary.- OBJECTIVES: To evaluate the effect of age in morbidity-mortality and outcomes of the surgical treatment of benign prostatic hyperplasia (BPH).

METHOD: We performed a retrospective analysis of the medical records of 305 patients over 70 years undergoing surgery for BPH between 1999-2003, grouped in four categories depending on ages $70-80$ years and 
over 80, and type of surgery (transurethral resection or open prostatectomy). The variables evaluated were: American society of anesthesiologists (ASA) operative risk classification, surgical indication, preoperative factors, postoperative outcome and follow-up. Statistical analysis was carried out with the Anova and chi-square tests Ip $=0.051$.

RESULTS: Open prostatectomy was performed in 59.1\% of the cases and transurethral resection in the other $40.9 \%$. 98.4\% of the patients showed some degree of comorbidity. Indwelling transurethral catheter was the main clinical feature of patients undergoing surgery over 80 years of age; the main characteristic in patients between 70-80 years was the existence of severe symptoms. Urological complications appeared more frequently than general complications (14\% vs $10.1 \%$ ). The most frequent urological complications were mild, mainly inflammatory-infectious diseases. Urological and general complications were more frequently observed in the open prostatectomy group, with no differences between ages. Patient satisfaction was high in the four groups (84.8\%-96.2\%). No case of perioperative mortality happened and only one patient died in the immediate post operative period.

CONCLUSIONS: We think the differences observed cannot be attributed to chronological age as clinical risk factor. Therefore, these patients could benefit of a definitive surgical treatment which will improve their quality of life.

Keywords: Benign prostatic hyperplasia. Age. Comorbidity.

\section{INTRODUCCIÓN}

Mientras que el siglo XX se ha caracterizado por el crecimiento de la población, el siglo XXI será el del envejecimiento de la misma como consecuencia de la inversión de la pirámide poblacional. El aumento en la expectativa de vida junto con la exigencia de una vida de calidad, supone un aumento en la demanda sanitaria y asistencial en el anciano. Por lo tanto, ya que la hiperplasia benigna de próstata (HBP) es una patología frecuente e inherente a la edad estamos asistiendo a un cambio en el patrón demográfico del paciente urológico. El efecto de la edad así como de las comorbilidades en la morbimortalidad perioperatoria en el tratamiento quirúrgico de la HBP ha sido ampliamente debatido. Con este estudio se pretende valorar el efecto de la edad de los pacientes en la morbi-mortalidad y en el resultado del tratamiento quirúrgico de la HBP.

\section{MATERIAL Y MÉTODO}

Se ha realizado un estudio retrospectivo de 336 pacientes intervenidos por HBP en el Servicio de Urología del Hospital Universitario Dr. Peset de Valencia durante el periodo comprendido entre enero 1999 y diciembre 2003. Se han diferenciado 4 grupos de pacientes en función de la edad y tipo de intervención realizada.

El grupo 1 constituido por aquellos pacientes mayores de 80 años sometidos a cirugía abierta. El grupo 2 formado por pacientes mayores de 80 años a los que se ha realizado una resección transuretral de próstata (RTU próstata). El grupo 3 integrado por pacientes de edades comprendidas entre 70 y 80 años de edad y cirugía abierta de la HBP, y el grupo 4 con pacientes de entre 70 y 80 años de edad y RTU de próstata. El criterio de inclusión utilizado para seleccionar la muestra fue el de pacientes mayores de 70 años de edad con diagnóstico histológico de HBP tras la intervención quirúrgica, apareciendo como motivos de exclusión la histología compatible con neoplasia de próstata, aquella patología relacionada con la interferencia en el vaciado vesical diferente de la HBP y la no disponibilidad de la historia clínica por estar microfilmada, obteniendo finalmente una muestra $\mathrm{n}=305$ pacientes.

Al mismo tiempo ha sido necesario estratificar 5 niveles de estudio según:

- la co-morbilidad preoperatoria (ASA);

- la indicación quirúrgica (sondaje permanente, prostatismo severo, hematuria, y litiasis vesical);

- factores relacionados con la cirugía atendiendo a tipo de anestesia utilizada, tiempo quirúrgico empleado, necesidad de transfusión de hemoderivados (>1 bolsa), y el éxitus peroperatorio del paciente;

- evolución postoperatoria donde se han recogido las complicaciones generales y urológicas, la necesidad de transfusiones (>1 bolsa), la estancia postoperatoria (días) y los éxitus;

- y el resultado en el seguimiento (meses) al alta hospitalaria contemplando el nivel de satisfacción, entendida como la presencia de micciones espontáneas con buen calibre del chorro de la orina sin incontinencia a los 3 meses de la cirugía, la aparición de complicaciones y la necesidad de reintervención.

Como método estadístico se ha utilizado el test Anova para la media y desviación estandar, y el test $\chi^{2}$ con la corrección de Yates para las proporciones, con un intervalo de confianza del $95 \%$. 


\section{RESULTADOS}

El grupo 1 está constituido por 23 pacientes con edad media de 82.13+3.08 [80-87]. El grupo 2 lo forman 26 pacientes con una edad media de $81.8+2.19$ [80-87]. El grupo 3, 157 pacientes de edad media 74.08+3.08 [70-79], y el grupo 4, 99 pacientes edad media 73.70+2.64 [70-79].

La comorbilidad preoperatoria según el ASA para cada grupo se distribuye de la siguiente forma. Para la cirugía abierta, dentro del grupo 1 no se encontraron niveles de ASA I ni IV siendo 9 pacientes (39.1\%) ASA II y 14 pacientes (60.9\%) ASA III. En el grupo 3, el criterio ASA I lo cumplían 3 pacientes (1.9\%), ASA II 91 pacientes $(58.0 \%)$, ASA III 62 pacientes $(39.2 \%)$ y 1 paciente $(0.6 \%)$ se clasificó como ASA IV. Siendo estadísticamente significativa la diferencia encontrada en el nivel de ASA III para el grupo 1 y $3, p=0.049$. Para la RTU de próstata en el grupo 2, un paciente $(3.8 \%)$ era ASA I, 11 pacientes (42.3\%) ASA II, 13 pacientes $(50 \%)$ ASA III, y un paciente $(3.8 \%)$ ASA IV. Los pacientes del grupo 4 se distribuían en uno (1\%) ASA I, 49 pacientes (49.5\%) ASA II, 46 pacientes (46.5\%) ASA III, y 3 pacientes (3\%) ASA IV (Tabla I).

El sondaje vesical permanente fue la indicación quirúrgica más frecuente para el grupo de mayor edad tanto en cirugía abierta como RTU próstata, con 13 pacientes $(56.5 \%)$ en el grupo 1 y 62 pacientes $(39.2 \%)$ en el grupo $3 ; 11$ pacientes $(42.3 \%)$ en el grupo 2 y 19 pacientes (19.2\%) en el grupo 4 $(p=0.014)$.

El prostatismo severo constituyó la indicación quirúrgica más frecuente en el grupo de menor edad para ambas técnicas siendo la diferencia con los pacientes más añosos significativa, 8 pacientes en el grupo $1(34.8 \%)$ y 94 pacientes $(59.5 \%)$ en el grupo 3 con una diferencia estadísticamente significativa $p=0.025$; 15 pacientes (17.7\%) en el grupo 2 y 75 pacientes $(75.8 \%)$ en el grupo 4 con una diferencia significativa $\mathrm{p}=0.001$. En el grupo 1, 6 pacientes $(26.1 \%)$ fueron intervenidos por hematuria persistente 0 intensa y 23 pacientes (14.6\%) en el grupo 3. Debido a la presen-

TABLA I. COMORBILIDAD PREOPERATORIA.

\begin{tabular}{|lcccccc|}
\hline & Grupo 1 & Grupo 3 & & Grupo 2 & Grupo 4 & \\
& $\mathrm{N}: 23$ & $\mathrm{~N}: 157$ & $\mathrm{~N}: 26$ & $\mathrm{~N}: 99$ & \\
\hline ASA I & - & $3(1.9 \%)$ & & $1(3.8 \%)$ & $1(1 \%)$ & \\
\hline ASA II & $9(39.1 \%)$ & $91(58.0 \%)$ & pns & $11(42.3 \%)$ & $49(49.5 \%)$ & $\mathrm{pns}$ \\
\hline ASA III & $14(60.9 \%)$ & $62(39.2 \%)$ & $\mathrm{p}=0.05$ & $13(50 \%)$ & $46(46.5 \%)$ & $\mathrm{pns}$ \\
\hline ASA IV & - & $1(0.6 \%)$ & & $1(3.8 \%)$ & $3(3 \%)$ & \\
\hline
\end{tabular}

TABLA II. INDICACIÓN QUIRÚRGICA.

\begin{tabular}{|lcccccc|}
\hline & Grupo 1 & Grupo 3 & & Grupo 2 & Grupo 4 & \\
& $\mathrm{N}: 23$ & $\mathrm{~N}: 157$ & & $\mathrm{~N}: 26$ & $\mathrm{~N}: 99$ & \\
\hline Sonda permanente & $13(56.5 \%)$ & $62(39.2 \%)$ & $\mathrm{pns}$ & $11(42.3 \%)$ & $19(19.2 \%)$ & $\mathrm{p}=0.014$ \\
\hline Prostatismo severo & $8(34.8 \%)$ & $94(59.5 \%)$ & $\mathrm{p}=0.025$ & $15(17.7 \%)$ & $75(75.8 \%)$ & $\mathrm{p}=0.001$ \\
\hline Hematuria & $6(26.1 \%)$ & $23(14.6 \%)$ & pns & $1(3.8 \%)$ & $17(17.2 \%)$ & $\mathrm{pns}$ \\
\hline Litiasis vesical & $5(21.7 \%)$ & $12(7.6 \%)$ & pns & $2(7.7 \%)$ & $4(4 \%)$ & $p n s$ \\
\hline
\end{tabular}


cia de litiasis vesical se indicó tratamiento quirúrgico en 5 pacientes $(21.7 \%)$ del grupo 1 y 12 pacientes $(7.6 \%)$ del grupo 3. Por hematuria se intervino un paciente $(3.8 \%)$ del grupo 2 y 17 pacientes $(17.2 \%)$ del grupo 4 y por litiasis vesical 2 pacientes $(7.7 \%)$ del grupo 2 y 4 pacientes (4\%) del grupo 4 (Tabla II).

La anestesia general fue utilizada en 17 pacientes $(73.9 \%)$ del grupo 1 y 134 pacientes (85.3\%) del grupo 3. La anestesia regional fue la elección en 6 pacientes $(26.1 \%$ ) del grupo 1 y 23 pacientes $(14.6 \%)$ del grupo 3 . El tiempo de cirugía se contabilizó en $62.8+13.3 \mathrm{~min}$ en el grupo 1 y $74.2+21.29 \mathrm{~min}$ en el grupo 3. En las RTU próstata sólo se utilizó anestesia general en 11 pacientes (11.1\%) del grupo 4 y anestesia regional en todos los pacientes del grupo 2 y 88 pacientes $(88.9 \%)$ del grupo 4. El tiempo empleado para realizar la RTU próstata fue de $51.5+13.6$ minutos en el grupo 2 y $49.5+12.62$ minutos en el grupo 4 tratándose de la única diferencia estadísticamente significativa hallada en este nivel de estudio. En ninguno de los grupos aconteció ningún éxitus intraoperatorio (Tabla III).

Las complicaciones urológicas, generales y en el seguimiento tras el alta hospitalaria vienen recogidas en la Tabla IV según su naturaleza, porcentaje de aparición y grupo de pacientes.

Las complicaciones urológicas y generales aparecieron en un $17.4 \%$ y $13.04 \%$ respectivamente en el grupo $1,22.9 \%$ y $12.7 \%$ en el grupo 3 , sin encontrar diferencias estadísticamente significativas. Dentro del grupo 2 las complicaciones urológicas y generales se dieron por igual $(7.6 \%)$, y en el grupo 4 recogemos un $3.03 \%$ de complicaciones urológi- cas y un $4.04 \%$ de complicaciones generales, sin encontrar diferencias significativas entre los diferentes grupos. La estancia media para el grupo 1 fue de 11.09+9.44 días, para el grupo 3 de $8.9+3.90$ días, para el grupo 2 de $5.4+1.80$ días y para el grupo 4 de $3.98+1.47$ días encontrando en este último caso la única diferencia estadísticamente significativa $(p=0.001)$. Sólo tuvimos un éxitus postoperatorio de un paciente perteneciente al grupo 3 (Tabla V).

En el último nivel de estudio que atiende al seguimiento tras la intervención quirúrgica se han recogido 2 complicaciones (8.6\%) en el grupo 1, 32 complicaciones $(20.4 \%)$ en el grupo 3 , una complicación (3.8\%) en el grupo 2 y $15(15.5 \%)$ en el grupo 4 sin encontrar diferencias estadísticamente significativas para cada grupo de edad según el tipo de intervención. El nivel de satisfacción fue del $87.0 \%$ en el grupo 1,89.1\% en el grupo 3, $96.2 \%$ en el grupo 2, y $84.8 \%$ en el grupo 4 . Se reintervinieron 3 pacientes $(1.9 \%)$ del grupo 3 y un paciente $(1 \%)$ del grupo 4 por esclerosis de cuello. El seguimiento medio es de $7.85+6.99$ meses en el grupo $1,11.4+11.28$ en el grupo 3, 9.7+12.8 en el grupo 2, y $17.3+16.9$ en el grupo 4 con una diferencia estadísticamente significativa entre el grupo 2 y 4 ( $p=0.033$ ) (Tabla VI).

Al valorar los grupos de edad distribuidos según la misma comorbilidad ASA no se han encontrado diferencias estadísticamente significativas en la aparición de complicaciones urológicas, complicaciones generales y complicaciones en el seguimiento tras la intervención, así como la estancia media hospitalaria, ni en los que se realizó cirugía abierta ni en los que se realizó RTU próstata (Tabla VII).

TABLA III. FACTORES RELACIONADOS CON LA CIRUGÍA.

\begin{tabular}{|lcccccc|}
\hline & Grupo 1 & Grupo 3 & & Grupo 2 & Grupo 4 & \\
& $\mathrm{N}: 23$ & $\mathrm{~N}: 157$ & & $\mathrm{~N}: 26$ & $\mathrm{~N}: 99$ & \\
\hline Anestesia general & $17(73.9 \%)$ & $134(85.3 \%)$ & pns & - & $11(11.1 \%)$ & \\
\hline Anestesia regional & $6(26.1 \%)$ & $23(14.6 \%)$ & pns & $26(100 \%)$ & $88(88.9 \%)$ & pns \\
\hline Tiempo de cirugía & $62.8 \pm 13.3$ & $74.2 \pm 21.29$ & pns & $51.5 \pm 13.6$ & $49.5 \pm 12.62$ & p:0.015 \\
\hline Transfusión & $5(21.17 \%)$ & $24(15.2 \%)$ & & - & $2(2 \%)$ & \\
\hline Éxitus & - & - & & - & & \\
\hline
\end{tabular}




\section{DISCUSIÓN}

Nuestro porcentaje de cirugía abierta, $59.1 \%$ de cirugía abierta vs. $40.9 \%$ de RTU próstata, es mayor que el de las publicaciones revisadas. Como en la literatura, un elevado número de pacientes presen- tan comorbilidades $(1,2)$ y se clasifican como ASA II, III y IV, sólo 5 pacientes (1.6\%) catalogados como ASA I están libres de enfermedades asociadas. De nuestros resultados se desprende que a mayor edad mayor proporción de ASA III, aunque sólo se encuentra una diferencia estadísticamente significativa para

\section{TABLA IV. COMPLICACIONES TRAS LA CIRUGÍA.}

\begin{tabular}{|c|c|c|c|c|c|}
\hline \multicolumn{6}{|c|}{ Complicaciones urológicas } \\
\hline & Grupo 1 & Grupo 2 & Grupo 3 & Grupo 4 & Total \\
\hline Infección herida & $2(8.7 \%)$ & - & $15(9.5 \%)$ & - & $17(5.5 \%)$ \\
\hline Orquiepididimitis & $1(4.3 \%)$ & - & $7(4.4 \%)$ & - & $8(2.6 \%)$ \\
\hline Fístula urinaria & $1(4.3 \%)$ & - & $4(2.5 \%)$ & - & $5(1.6 \%)$ \\
\hline Sépsis & - & - & $2(1.2 \%)$ & $1(0.01 \%)$ & $3(0.1 \%)$ \\
\hline Serosa pared & - & - & $5(3.1 \%)$ & - & $5(1.6 \%)$ \\
\hline Hematuria+transfusión & - & $1(3.8 \%)$ & $5(3.1 \%)$ & - & $6(1.9 \%)$ \\
\hline Taponamiento celda & $1(4.3 \%)$ & - & $1(0.6 \%)$ & - & $2(0.6 \%)$ \\
\hline Sdr. Reabsorción & - & $1(3.8 \%)$ & - & $2(0.02 \%)$ & $3(0.1 \%)$ \\
\hline \multicolumn{6}{|c|}{ Complicaciones generales } \\
\hline & Grupo 1 & Grupo 2 & Grupo 3 & Grupo 4 & Total \\
\hline Cardiovasculares & - & - & $3(1.9 \%)$ & $1(1 \%)$ & $4(1.3 \%)$ \\
\hline Cerebrovasculares & $1(4.3 \%)$ & - & $1(0.6 \%)$ & $1(1 \%)$ & $3(0.9 \%)$ \\
\hline Neumonía & - & $1(3.8 \%)$ & $2(1.2 \%)$ & $1(1 \%)$ & $4(1.3 \%)$ \\
\hline CID & $1(4.3 \%)$ & - & $2(1.2 \%)$ & - & $3(0.9 \%)$ \\
\hline I.Renal aguda & $2(8.6 \%)$ & - & $4(2.5 \%)$ & - & $6(1.9 \%)$ \\
\hline TVP & - & - & $2(1.2 \%)$ & - & $2(0.6 \%)$ \\
\hline Sdr. Confusional & - & $1(3.8 \%)$ & $4(2.5 \%)$ & - & $5(1.6 \%)$ \\
\hline Otras & - & - & $4(2.5 \%)$ & $1(1 \%)$ & $5(1.6 \%)$ \\
\hline \multicolumn{6}{|c|}{ Complicaciones en la evolución al alta hospitalaria } \\
\hline & Grupo 1 & Grupo 2 & Grupo 3 & Grupo 4 & Total \\
\hline ITU & - & - & $10(6.3 \%)$ & $7(7 \%)$ & $17(5.5 \%)$ \\
\hline Fístula urinaria & - & - & $2(1.2 \%)$ & - & $2(0.6 \%)$ \\
\hline Orquiepididimitis & - & - & $9(5.7 \%)$ & $1(1 \%)$ & $10(3.2 \%)$ \\
\hline Esclerosis meato & - & - & $3(1.9 \%)$ & $3(3 \%)$ & $6(1.9 \%)$ \\
\hline Esclerosis cuello & - & - & $3(1.9 \%)$ & $1(1 \%)$ & $4(1.3 \%)$ \\
\hline Estenosis uretra & $1(4.3 \%)$ & - & - & $3(3 \%)$ & $4(1.3 \%)$ \\
\hline Incontinencia & - & - & - & - & $2(0.6 \%)$ \\
\hline Hematuria & $1(4.3 \%)$ & $1(0.6 \%)$ & $1(0.6 \%)$ & $1(1 \%)$ & $4(1.3 \%)$ \\
\hline Eventración & - & - & - & - & $2(0.6 \%)$ \\
\hline
\end{tabular}


el grupo de cirugía abierta, grupo 1 y 3, con comorbilidad ASA III.

Al igual que recogen otros autores, la indicación quirúrgica más frecuente en los pacientes de mayor edad la establece la aparición de alguna complicación del prostatismo (retención aguda de orina y sonda permanente) a diferencia del prostatismo severo que es la indicación quirúrgica más frecuente en el grupo de pacientes de 70-80 años de edad $(2,3,4)$. Se podría pensar entonces, como refiere Ibrahim y cols., que pudieran acontecer más complicaciones urológicas en pacientes intervenidos de mayor edad, sin embargo nuestros resultados no lo confirman.

En estos pacientes la elección del tipo de cirugía se estableció fundamentalmente según el tamaño del adenoma, peso estimado en gramos, y la habilidad del cirujano. En un $73.9 \%$ y $85.3 \%$ de los casos de cirugía abierta, grupo 1 y 3 respectiva- mente, se utilizó anestesia general y en el $100 \%$ y $88.9 \%$ de los casos de RTU de próstata, grupo 2 y 4 respectivamente, se llevó a cabo anestesia regional sin encontrar diferencias estadísticamente significativas para los grupos de edad. De acuerdo con la literatura (3) mayor proporción de pacientes requirió transfusión de hemoderivados en los grupos 1 y 3 atribuible a la técnica quirúrgica. Se encuentra una diferencia significativa en un mayor tiempo quirúrgico para las RTU de próstata de los pacientes mayores de 80 años respecto a los de 70-80 años, que podría tener relación con un adenoma de mayor tamaño en los pacientes de mayor edad, como señala Pientka y cols.(2).

Las complicaciones urológicas en el postoperatorio inmediato, en concreto las menos graves, sobretodo inflamatorio-infecciosas (infección de la herida quirúrgica, orquiepididimitis, fístula urinaria cutánea, seroma de pared, hematuria), se presentan

\section{TABLA V. EVOLUCIÓN POSTOPERATORIA.}

\begin{tabular}{|lcccccc|}
\hline & Grupo 1 & Grupo 3 & & Grupo 2 & Grupo 4 & \\
& $\mathrm{N}: 23$ & $\mathrm{~N}: 157$ & & $\mathrm{~N}: 26$ & $\mathrm{~N}: 99$ & \\
\hline Complic. urológicas & $4(17.4 \%)$ & $36(22.9 \%)$ & pns & $2(7.6 \%)$ & $3(3.03 \%)$ & \\
\hline Complic. generales & $3(13.04 \%)$ & $20(12.7 \%)$ & pns & $2(7.6 \%)$ & $4(4.04 \%)$ & pns \\
\hline Transfusiones & $3(13.3 \%)$ & $23(14.6 \%)$ & pns & $1(3.8 \%)$ & $1(1 \%)$ & \\
\hline Estancia media & $11.0 \pm 9.44$ & $8.9 \pm 3.90$ & pns & $5.4 \pm 1.80$ & $3.98 \pm 1.47$ & p:0.001 \\
\hline Éxitus & - & $1(0.6 \%)$ & & - & - & \\
\hline
\end{tabular}

TABLA VI. SEGUIMIENTO.

\begin{tabular}{|lcccccc|}
\hline & Grupo 1 & Grupo 3 & & Grupo 2 & Grupo 4 & \\
& $\mathrm{N}: 23$ & $\mathrm{~N}: 157$ & & $\mathrm{~N}: 26$ & $\mathrm{~N}: 99$ & \\
\hline Complicaciones & $2(8.6 \%)$ & $32(20.4 \%)$ & pns & $1(3.8 \%)$ & $15(15.5 \%)$ & pns \\
\hline Satisfacción & $20(87.0 \%)$ & $140(89.1 \%)$ & pns & $25(96.2 \%)$ & $84(84.8 \%)$ & pns \\
\hline Reintervención & - & $3(1.9 \%)$ & & - & $1(1 \%)$ & \\
\hline (x \pm sd) & $7.85 \pm 6.99$ & $11.4 \pm 11.28$ & pns & $9.7 \pm 12.8$ & $17.3 \pm 16.9$ & p:0.033 \\
\hline
\end{tabular}


con más frecuencia que las complicaciones generales que oscilan entre el $0.6 \%$ y el $1.9 \%$. Las complicaciones urológicas y generales aparecen sobre todo en el grupo de cirugía abierta sin encontrar diferencias significativas según el grupo de edad. Tampoco se encuentran diferencias en la necesidad de hemoderivados, observando que la transfusión se da más en la cirugía abierta independientemente de la edad coincidiendo con la literatura (3). Aunque se aprecia en los resultados que la estancia media postoperatoria es mayor en los pacientes de mayor edad para ambos tipos de cirugía, tan solo se encuentra una diferencia estadísticamente significativa a favor de una mayor estancia media para los pacientes mayores de 80 años con RTU de próstata vs los pacientes de 70-80 años con RTU de próstata.

Para Ibrahim y cols., la comorbilidad y nunca la edad de los pacientes tratados por HBP con cirugía abierta se asocia tan solo, a diferencia de otros como Pientka y Mebust, con una estancia postoperatoria prolongada siendo que en los demás aspectos ninguna de las dos circunstancias, comorbilidad y edad, influye en los resultados de la cirugía abierta de la $\operatorname{HBP}(2,3,5)$. Pientka y cols. concluye que la edad no es un factor de riesgo en la aparición de complicaciones tras la RTU de próstata (2). En un estudio retrospectivo de 60 meses de seguimiento medio de pacientes mayores de 80 años intervenidos de RTU de próstata, se registraba una comorbilidad aceptable y una tasa de supervivencia similar para ese grupo de edad en la población general (1). Aunque, a diferencia de nuestros resultados, para Krogh y cols. (6) los pacientes mayores de 80 años a los que se había realizado una RTU de próstata presentan más complicaciones menores y corregibles, tampoco se encuentran diferencias en la morbi-mortalidad y el resultado de la cirugía, por lo que para él la edad no influye de forma importante en el resultado del tratamiento quirúrgico mediante RTU de próstata. Por otro lado Nadu y cols. (4) encuentra más complicaciones, sobre todo mayores, en pacientes ancianos, y Wyatt y cols. (7) encuentra también una morbilidad elevada recomendando una detallada valoración preoperatoria del paciente. No tenemos mortalidad perioperatoria, siendo la que se recoge en la literatura del orden de $1.3 \%-2.1 \%(1,7)$, y sólo ocurrió un éxitus en un paciente del grupo 3 .

La proporción de pacientes reintervenidos, sólo en las RTU de próstata, es baja y comparable a la recogida en la literatura, siendo ligeramente ma-

TABLA VII. SUBGRUPOS DE PACIENTES DE DIFERENTE EDAD HOMOGÉNEOS PARA TIPO DE CIRUGÍA Y ASA.

\begin{tabular}{|c|c|c|c|c|c|c|}
\hline \multicolumn{7}{|l|}{ CIRUGÍA ABIERTA } \\
\hline & ASA II & ASA II & & ASA III & ASA III & \\
\hline Complicaciones urológicas & $19(20.87 \%)$ & $1(11.1 \%)$ & pns & $15(24.2 \%)$ & $4(28.57 \%)$ & pns \\
\hline Complicaciones generales & $11(12.08 \%)$ & $2(22.2 \%)$ & pns & $11(17.74 \%)$ & $2(14.88 \%)$ & pns \\
\hline Complicaciones alta hospitalaria & $21(23.1 \%)$ & - & & $12(19.35 \%)$ & $2(14.88 \%)$ & \\
\hline Estancia & $8.42 \pm 2.42$ & $8.89 \pm 4.07$ & pns & $9.23 \pm 4.27$ & $12.43 \pm 11.63$ & pns \\
\hline \multicolumn{7}{|l|}{ RTU PRÓSTATA } \\
\hline & ASA \| & ASA II & & ASA III & ASA III & \\
\hline Complicaciones urológicas & $1(2.04 \%)$ & $1(9.09 \%)$ & & $1(2.7 \%)$ & $1(7.69 \%)$ & \\
\hline Complicaciones generales & $1(2.04 \%)$ & $1(9.09 \%)$ & & $4(8.69 \%)$ & $1(7.69 \%)$ & pns \\
\hline Complicaciones alta hospitalaria & $10(20.4 \%)$ & $1(9.09 \%)$ & pns & $7(15.21 \%)$ & - & \\
\hline Estancia & $3.7 \pm 0.84$ & $5.55 \pm 2.25$ & pns & $4.02 \pm 1.5$ & $5.38 \pm 1.55$ & p:0.005 \\
\hline
\end{tabular}


yor para Matani y cols. con un $4.2 \%(1,7,8)$. Presentando como complicaciones en el seguimiento en la consulta, las infecciosas principalmente siendo baja la proporción de esclerosis celda, estenosis de uretra e incontinencia en un $1.3 \%, 1.3 \%$ y $0.6 \%$ respectivamente.

Por último, cabe destacar que para un seguimiento medio prolongado la satisfacción es elevada en todos los grupos $(84.8 \%-96.2 \%)$, coincidiendo con las publicaciones revisadas (7-10).

\section{CONCLUSIÓN}

Valorando grupos de pacientes de diferente edad, homogéneos en cuanto al tipo de cirugía y la comorbilidad preoperatoria ASA, no se encuentran diferencias significativas en la aparición de complicaciones, urológicas, generales y en el seguimiento, ni en la estancia media postoperatoria. Con un nivel de satisfacción elevado tras el tratamiento quirúrgico.

Por lo tanto las diferencias previas observadas pueden ser debidas a otras variables y no a la edad cronológica per se entendida como factor de riesgo clínico, pudiendo beneficiarse estos pacientes de un tratamiento quirúrgico resolutivo que mejore su calidad de vida, en lugar del "watchful waiting" condenándolos a una sonda permanente $o$ a la limitante sintomatología de un prostatismo severo.

\section{BIBLIOGRAFÍA y LECTURAS RECOMENDADAS (*lectura de interés $y^{* *}$ lectura fundamental)}

**1. MATANI, Y.; MOTTRIE AM, STOCKLE M y cols. "Transurethral prostatectomy: a long-term follow-up study of 166 patients over 80 years of age.” Eur. Urol.; 30:414, 1996.

**2. PIENKA, L.; VAN LOGHEM, J.; HAHN, E. y cols. "Comorbidities and perioperative complications among patients with surgically treated benign prostatic hyperplasia" Urology; 38 supl.:43, 1991.

**3. IBRAHIM, A.; EL-MALIK, E.; GHALI, M. y cols. "Effect of age, comorbidity and type of surgery on perioperative complications and mortality of prostatectomy" BJUrol; 76:341, 1995.

4. NADU, A., MABJEESH, N.J.; BEN-CHAIM, J. $\mathrm{y}$ cols. "Are indications for prostatectomy in octogenarians the same as for younger men?" Int. Urol. Nephrol; 36:47, 2004.

5. MEBUST, W.K.; HOLTGREWE, H.L.; COCKETT, A.T.K. y cols. "Transurethral prostatectomy: immediate and postoperative complications. A cooperative study of 13 participating institutions evaluating 3885 patients" J. Urol.;141:243, 1989.

*6. KROGH, J.; JENSEN, J.S.; IVERSEN, H.G.; y cols. "Age as a prognostic variable in patients undergoing transurethral prostatectomy" Scand. J.

* Urol. Nephrol; 27:225, 1993.

7. WYATT, M.G.; STOWER, M.J.; SMITH, P.J.B. y cols. "Prostatectomy in the over 80-year-old" B. J. Urol.;64:417, 1989.

8. HABIB, N.A.; LUCK, R.J.: "Results of transurethral resection of benign prostate" Br. J. Surg.; 70:218, 1983.

9. ILKAER, L.B.; LUND, L.; NIELSEN, K.T. "Outcome of transurethral prostatectomy in men over 80 years". Scand. J. Urol. Nephrol.;32:270, 1998.

*10. LUTTWAK, Z., LASK, D.; ABARBANEL, J. y cols. "Transvesical prostatectomy in elderly patients" J. Urol.;157:2210, 1997. 